\title{
Urethral stricture and urethritis in men in Scotland
}

\author{
A McMillan, M Pakianathan, J H Mao, C C A Macintyre
}

Genitourinary

Medicine Unit,

Department of

Medicine, Edinburgh

Royal Infirmary

A McMillan

M Pakianathan

Medical Statistics

Unit, University of

Edinburgh

J H Mao

C C A Macintyre

Correspondence to:

Dr A McMillan

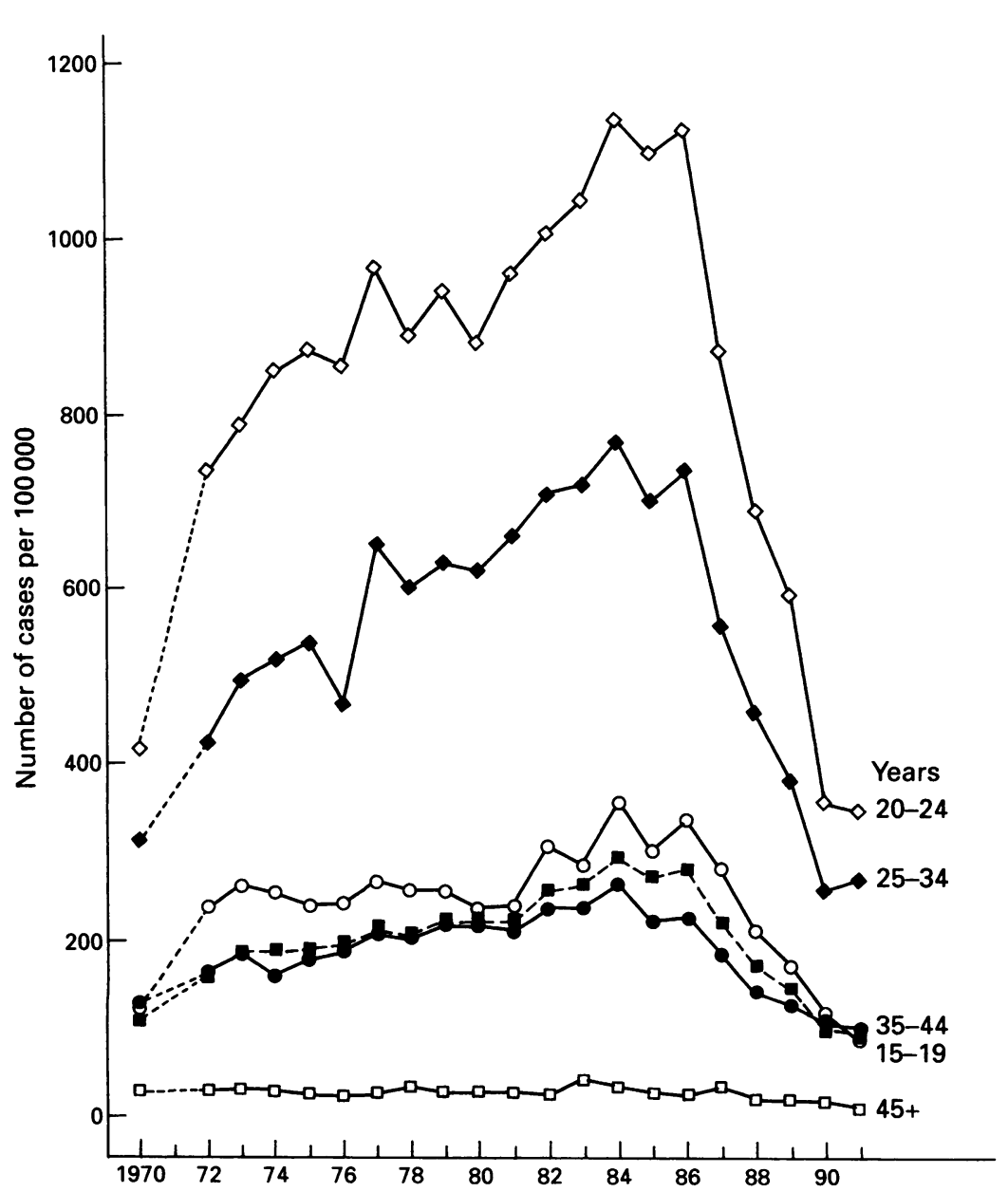

Figure 1 Number of cases of non-gonococcal urethritis in men per 100,000 population in

Figure 1 Number of cases of non-gonococcal uret
Scotland between 1970 and 1991. : all ages.

\begin{abstract}
Objectives-To examine the incidence of urethral stricture in men in Scotland during the years 1982-1991 in relation to the changing incidence of gonococcal and non-gonococcal urethritis (NGU) over the past 20 years.
\end{abstract}

Design-Retrospective study of incidence of urethral stricture in Scotland.

Method-The number of new men in whom a diagnosis of urethral stricture was made for the years 1982-1991 was obtained using the new Scottish Record Linkage system, and the number of cases of gonorrhoea and NGU was obtained from Communicable Diseases (Scotland) Unit. Age-specific rates of urethral stricture were calculated and the Poisson regression model was used to test if there was a trend of rate with age or time change. Results-There was a highly significant increase in the incidence of urethral stricture with age but only a slight increase in incidence over the study period within each age group.

Conclusion-As the interaction between age and time was not significant, it is concluded that urethritis associated with sexually transmitted organisms is an uncommon cause of urethral stricture in Scotland.

(Genitourin Med 1994;70:403-405)

\section{Introduction}

Until recently, non-gonococcal urethritis (NGU) was a condition that was diagnosed commonly in men who attended genitourinary medicine (GUM) clinics. In over 50\% of cases, NGU is associated with infection with the oculogenital serovars of Chlamydia trachomatis; the cause in the other cases is still uncertain, but mycoplasmas, ureaplasmas, anaerobic infections and, rarely, herpes simplex virus and Trichomonas vaginalis may have a role. ${ }^{1}$ Neisseria gonorrhoeae also causes urethritis, and it is of note that about $30 \%$ of men with urethral gonorrhoea have concurrent chlamydial infection. ${ }^{2}$

In the older text books of venereology, fibrous stricture of the urethra was recorded as a complication of inadequately treated gonorrhoea and of non-gonococcal urethritis (NGU) and in a urethroscopic study of men with urethritis who attended four sexually transmitted disease clinics in South East England, Dunlop ${ }^{3}$ found urethral stricture in almost $5 \%$ of 328 men with NGU and in nearly $2 \%$ of those with gonococcal urethritis (GU). Although the pathogenesis of urethral stricture associated with NGU is unknown, endemic trachoma caused by the trachoma serovars of $C$ trachomatis is known to be mediated by delayed hypersensitivity ${ }^{4}$ and that the chronic inflammation and scarring results from repeated exposure to chlamydial antigen either through reinfection or persistent infection. ${ }^{5}$

Symptomless infection of the uterine cervix with $C$ trachomatis is common amongst sexually active young women who attend GUM clinics and reinfection of the urethras of men who have had multiple sexual partners is well recognised. In addition, despite treatment of their sexual partners on epidemiological grounds, NGU recurs in $16 \%$ of men who have been treated for a first ever episode of chlamydial urethritis and in $27 \%$ of men treated for non-gonococcal, non-chlamydial urethritis. ${ }^{6}$ In some cases, this may result from re-exposure to chlamydiae but in many, the reasons for the poor clinical response to treatment and for recurrence are unknown. 


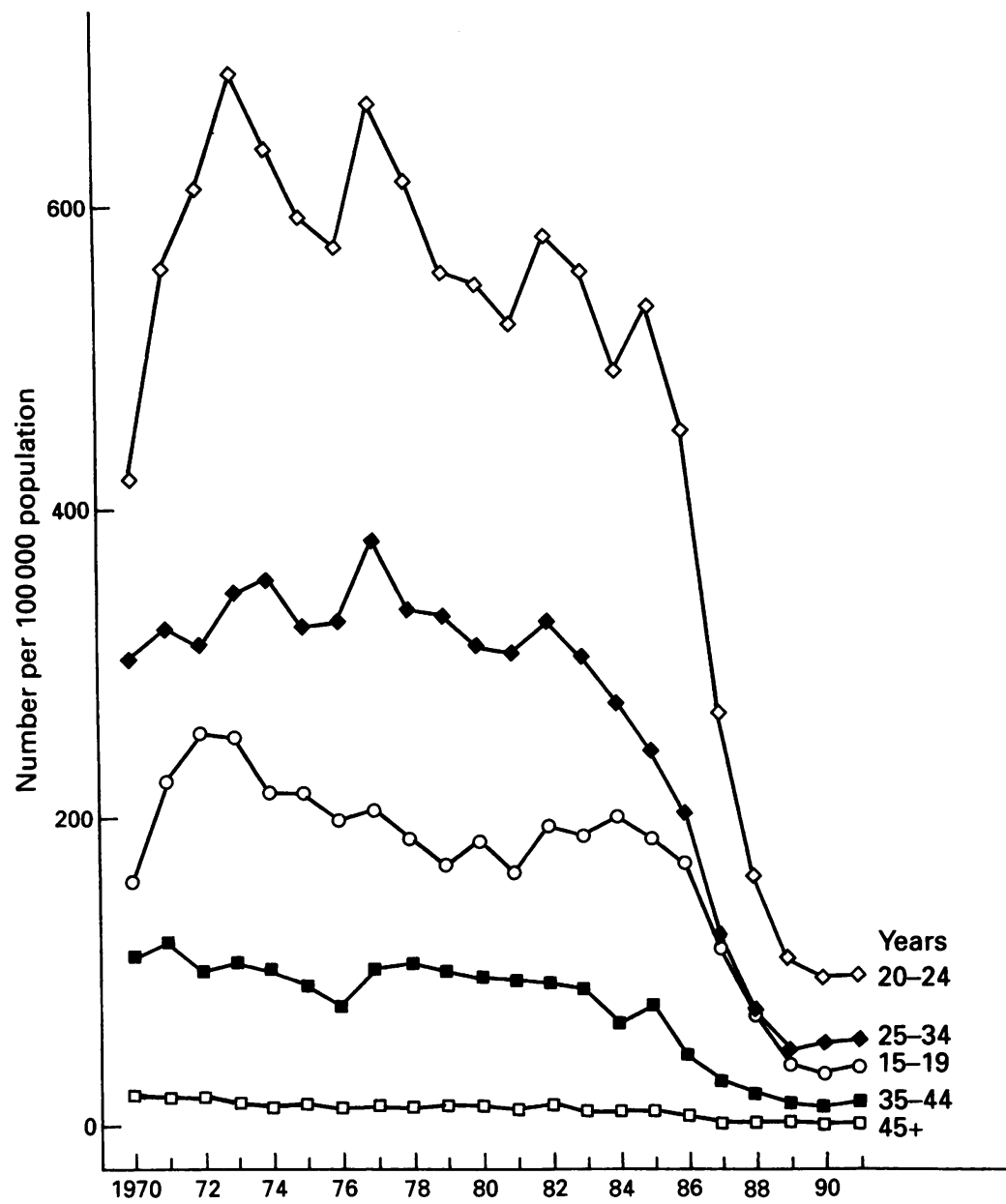

Figure 2 Number of cases of gonococcal urethritis in men per 100,000 population in Scotland between 1970 and 1991.

\section{Methods}

Data on the number of cases of NGU and GU reported annually from each genitourinary medicine clinic in Scotland from the years 1970 to 1991 inclusive were obtained from Communicable Diseases (Scotland) Unit, Ruchill Hospital, Glasgow. The number of new men in whom a diagnosis of urethral stricture was made for the years 1982 to 1991 inclusive, was obtained from the Information and Statistics Division, Directorate of Information Services, National Health Services in Scotland, using the Scottish Record Linkage System. This is a new system that has brought together records containing information about episodes of care on a patient basis ${ }^{7}$; unfortunately, linked data on the incidence of urethral stricture from previous years were unavailable.

Age-specific rates of urethral stricture per year were calculated and the Poisson regression model was used to test if there was a trend of rate with age or time change. The model is:

$\log _{e} n$ (number of cases) $=$

Constant $+\log _{\mathrm{e}} \mathrm{n}$ (population)

+ (effect due to age group)

+ (effect due to time period)

that is,

number of cases $=\exp \{$ Constant + (effect due to population $=$ age) + (effect due to time) $\}$

The use of this model allows for changes in age structure over time and produces comparable summary statistics over time. The model also allows one to investigate whether the changes over time vary between age groups (referred to as an interaction).

It was therefore of concern to us that the number of cases of urethral stricture resulting from chronic inflammation may increase in direct proportion to the increasing incidence of NGU that was noted in the 1970 s and early 1980s. This study was designed to examine the incidence of urethral stricture in Scotland over the past decade and to determine if there was any temporal relationship between the incidence of stricture in different age groups and that of NGU or gonorrhoea over the preceding 15 years.

\section{Results}

The incidence of non-gonococcal urethritis in all age groups increased progressively from 1970 and peaked in 1984, before declining rapidly (fig 1). During each year, the incidence was greatest in those aged between 20 and 24 years.

The incidence of gonococcal urethritis peaked in the early to mid 1970s gradually declining thereafter and showing a sudden fall

Table Estimates of effects and rate ratio by age and year of diagnosis on the incidence of urethral stricture

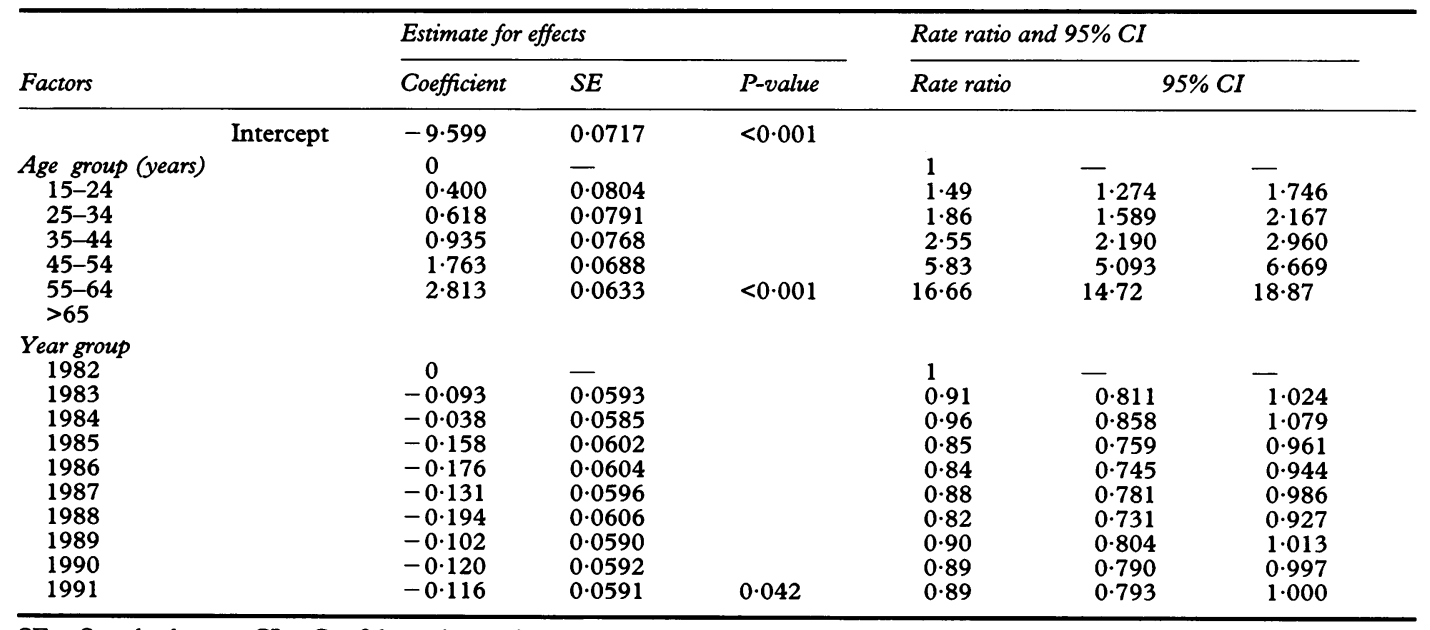

$\mathrm{SE}=$ Standard error. $\mathrm{CI}=$ Confidence interval. 


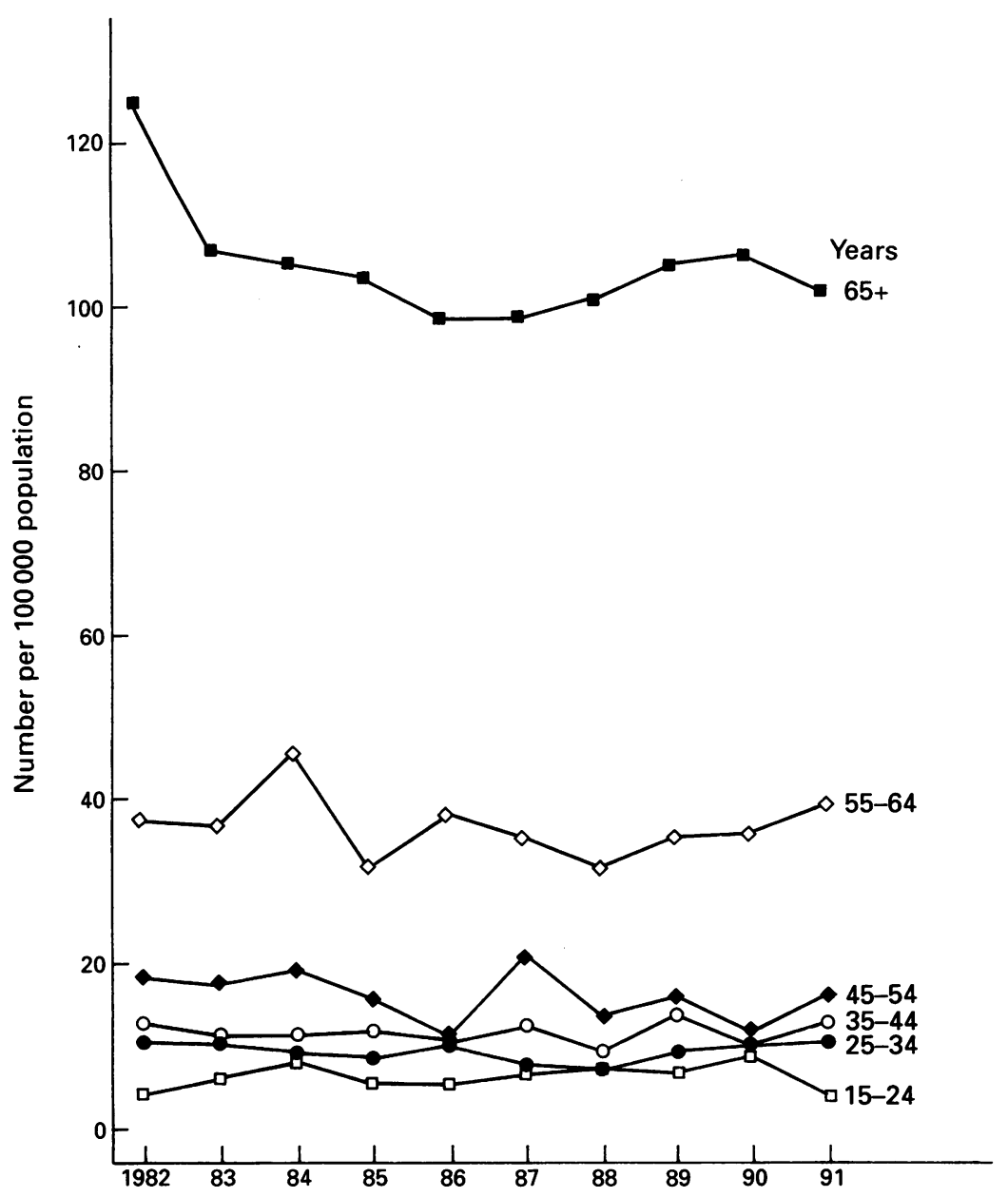

Figure 3 Number of cases of urethral stricture in men per 100,000 population in Scotland between 1982 and 1991.

in incidence from 1985 (fig 2); during each year, the incidence was greatest in those aged between 20 and 24 years.

Figure 3 shows the incidence of urethral stricture by age group and the table shows estimates of effects (coefficients); the exponent of coefficients gives the rate ratio, that is, the ratio of rates between each group compared with the baseline. The effect due to age was highly significant $(p<0.001)$, the incidence of stricture increasing with age. Although the effect due to time was just significant $(p=0.042)$ the interaction between age and time was not $(p>0.05)$.

\section{Discussion}

Urethral strictures may be congenital or acquired, the most common cause of the latter being trauma, either due to surgical instrumentation or pelvic fracture. Other rarer causes include tuberculosis and neoplasms. ${ }^{8}$ Unfortunately, information on the aetiology of the strictures in the patients reported here is not available.

Our data show clearly that the incidence of urethral stricture rises with age, a finding that is likely to reflect current surgical practice in the management of benign prostatic hyperplasia. Over the past 15 years, transurethral prostatectomy has been used increasingly in the management of this condition and urethral stricture is a well recognised complication of this procedure.
In Scotland the incidence of NGU increased progressively from the early 1970 s, peaked in 1984 and declined rapidly thereafter. The incidence of GU was maximal in the early $70 \mathrm{~s}$, showed a very gradual decline until 1985 and then suddenly fell. In both conditions, the highest incidence was noted in men aged between 20 and 24 years, although the overall numbers of cases of GU was significantly less than those of NGU.

If urethritis is an important cause of urethral stricture in young men, the incidence of this condition might have been expected to have risen from the early 1980s. Such an increase over the past decade was indeed noted, but this was slight and, importantly, the interaction between age and time was not significant. If the period before the development of the clinical features of urethral stricture after a sexually acquired urethritis is some 10 years $^{9}$ then one would have expected a disproportionate rise in incidence in the 35 to 44 year old age group. A possible explanation of any failure to identify a marked change with time in the incidence of stricture is the method of analysis that was based on only counting the earliest hospital episode with a main diagnosis of urethral stricture for a particular patient. This means that because the database went back only as far as 1982 , the figures in that year and possibly other years following were inflated by patients whose actual first diagnosis of urethral stricture occurred before 1982. The effect, however, is likely to be small. Another possibility for a failure to identify an increase in the incidence of urethral stricture is that it will not be noted until later. The time taken for the development of clinical features of stricture is somewhat uncertain but older studies suggest a prepatient period of some 10 years. ${ }^{9}$ There is one anecdotal report of a posterior urethral stricture developing within two and a half years of an episode of chlamydial urethritis. ${ }^{10}$

Our conclusion is that NGU and GU are uncommon causes of urethral stricture in Scotland, although the situation may be different in developing countries.

We acknowledge with gratitude the assistance given by members of staff of the Directorate of Information Services, Information and Statistics Division, Trinity Park House, Edinburgh.

1 Robertson DHH, McMillan A, Young H. Clinical Practice in Sexually Transmissible Diseases. 2nd edn, Edinburgh: Churchill Livingstone 1989.

2 Richmond SJ, Hilton AL, Clarke SKR. Chlamydial infection. Role of Chlamydia Subgroup A in non-gonococcal and post-gonococcal urethritis. Br $\mathcal{F}$ Venereal Dis 1972; and post-gon

3 Dunlop EMC. Incidence of urethral stricture in the male after urethritis. Br ₹ Venereal Dis 1961;37:64-9.

4 Watkins NG, Hadlow WJ, Moos AB, Caldwell HD. Ocular delayed hypersensitivity: a pathogenetic mechanism of chlamydial conjunctivitis in guinea pigs. Proc Natl Acad Sci 1986;83:7480-4.

5 Grayston JT, Wang S-L. New knowledge of chlamydiae and the diseases they cause. F Infect Dis 1975;132: 87-105.

6 Woolley PD, Wilson JD, Kinghorn GR. Epidemiological treatment of sexual contacts prevents recurrence of nongonococcal urethritis. Genitourin Med 1987;63:384-5.

7 Kendrick S, Clarke J. The Scottish Record Linkage System. Health Bull 1993:51:72-9.

8 Blandy JP. Urethral stricture. Postgrad Med f 1980;56: 383-418.

9 Kidd F (1910) cited in 8

10 Colleen S. cited in Oriel JD, Ridgway GL. Genital infection in men. Br Med Bull 1983;39:133-7. 